\title{
Maternal insulin sensitivity is associated with oral glucose-induced changes in fetal brain activity
}

\author{
Katarzyna Linder • Franziska Schleger • Caroline Ketterer • Louise Fritsche • \\ Isabelle Kiefer-Schmidt • Anita Hennige • Hans-Ulrich Häring • Hubert Preissl • \\ Andreas Fritsche
}

Received: 21 November 2013 / Accepted: 27 February 2014 / Published online: 28 March 2014

(C) Springer-Verlag Berlin Heidelberg 2014

\begin{abstract}
Aims/hypothesis Fetal programming plays an important role in the pathogenesis of type 2 diabetes. The aim of the present study was to investigate whether maternal metabolic changes during OGTT influence fetal brain activity.

Methods Thirteen healthy pregnant women underwent an OGTT $(75 \mathrm{~g})$. Insulin sensitivity was determined by glucose and insulin measurements at 0,60 and $120 \mathrm{~min}$. At each time point, fetal auditory evoked fields were recorded with a fetal magnetoencephalographic device and response latencies were determined.

Results Maternal insulin increased from a fasting level of $67 \pm 25 \mathrm{pmol} / 1$ (mean $\pm \mathrm{SD}$ ) to $918 \pm 492 \mathrm{pmol} / 160 \mathrm{~min}$ after glucose ingestion and glucose levels increased from $4.4 \pm 0.3$ to $7.4 \pm 1.1 \mathrm{mmol} / \mathrm{l}$. Over the same time period, fetal response
\end{abstract}

Katarzyna Linder and Franziska Schleger contributed equally to this work.

K. Linder · C. Ketterer · A. Hennige · H.-U. Häring · A. Fritsche Division of Endocrinology, Diabetology, Angiology, Nephrology and Clinical Chemistry, Department of Internal Medicine,

University Hospital Tübingen, Tübingen, Germany

K. Linder $\cdot$ F. Schleger $\cdot$ C. Ketterer $\cdot$ L. Fritsche $\cdot$ H.-U. Häring $•$ H. Preissl • A. Fritsche

German Center for Diabetes Research (DZD), Neuherberg, Germany

K. Linder $\cdot$ F. Schleger $\cdot$ C. Ketterer $\cdot$ L. Fritsche $\cdot$ H.-U. Häring $\cdot$

H. Preissl • A. Fritsche

Institute for Diabetes Research and Metabolic Diseases of the

Helmholtz Center Munich at the University of Tübingen (IDM),

Tübingen, Germany

F. Schleger $\cdot$ I. Kiefer-Schmidt $\cdot$ H. Preissl $(\bowtie)$

fMEG Center, University of Tübingen, Otfried-Müller-Str. 47,

72076 Tübingen, Germany

e-mail: hubert.preiss1@uni-tuebingen.de

I. Kiefer-Schmidt

Department of Obstetrics and Gynecology, University Hospital

Tübingen, Tübingen, Germany latencies decreased from $297 \pm 99$ to $235 \pm 84 \mathrm{~ms}(p=0.01)$ and then remained stable until $120 \mathrm{~min}(235 \pm 84 \mathrm{vs} 251 \pm 91 \mathrm{~ms}$, $p=0.39$ ). There was a negative correlation between maternal insulin sensitivity and fetal response latencies $60 \mathrm{~min}$ after glucose ingestion $(r=0.68, p=0.02)$. After a median split of the group based on maternal insulin sensitivity, fetuses of insulin-resistant mothers showed a slower response to auditory stimuli $(283 \pm 79 \mathrm{~ms})$ than those of insulin-sensitive mothers $(178 \pm 46 \mathrm{~ms}, p=0.03)$.

Conclusions/interpretation Lower maternal insulin sensitivity is associated with slower fetal brain responses. These findings provide the first evidence of a direct effect of maternal metabolism on fetal brain activity and suggest that central insulin resistance may be programmed during fetal development.

Keywords Brain insulin resistance $\cdot$ Fetal programming · Gestational diabetes
Abbreviations
AER Auditory evoked response
CTG Cardiotocography
ER Evoked response
fMEG Fetal magnetoencephalography

\section{Introduction}

The effects of insulin on the central nervous system play an important role in the pathogenesis of obesity and diabetes mellitus. Recently it has been suggested that changes in insulin action in the brain not only affect the regulation of appetite and body weight but are also crucial in controlling glucose metabolism [1-3]. There is evidence in humans that brain insulin resistance is not the consequence of peripheral metabolic changes but may itself contribute to the development of 
diabetes and obesity [4-6]. By recording brain activity with magnetoencephalography during a euglycaemichyperinsulinaemic stepwise clamp, we showed in adult humans that obesity, peripheral insulin resistance, age and genetic background are all associated with impaired central insulin sensitivity $[7,8]$.

The prevalence of obesity and type 2 diabetes mellitus is rising worldwide and the percentage of young people affected is increasing. The reasons for these changes are unclear, although environmental and epigenetic mechanisms are likely to be involved. A major epigenetic mechanism is the so-called fetal programming [9, 10]. Maternal environment and metabolism are of particular importance for the development of obesity and type 2 diabetes in offspring [11, 12]. Nutritional factors can alter fetal growth and glucose metabolism [13]. Furthermore, maternal metabolic changes related to type 2 diabetes or obesity influence the fetal phenotype [14]. Children of obese or diabetic mothers have an increased risk for type 2 diabetes and obesity in adulthood, independent of their genetic background $[15,16]$. Also, in fetuses of obese mothers peripheral insulin resistance is already developing in utero [17]. At present, it is unclear whether changes in maternal glucose metabolic status also have an influence on fetal brain activity.

Our aim was to study fetal brain activity during an OGTT in pregnant women. We hypothesised that the postprandial metabolism of the mother influences, or is associated with, fetal brain activity. Fetal magnetoencephalography (fMEG) enables us to non-invasively record brain activity in utero [18]. Fetal brain activity has mainly been studied as evoked activity to visual [19] and auditory stimulation [20, 21]. Response latencies to auditory stimuli decrease with fetal age and provide quantitative information on fetal functional brain maturation $[22,23]$. Therefore, in the present study we evaluated changes in fetal response latencies to auditory stimulation during the course of a routine OGTT in healthy, normal pregnancies. In addition, we determined correlations between fetal response latencies and maternal blood glucose and plasma insulin levels as indicators for the influence of maternal metabolic changes on fetal brain activity.

\section{Methods}

Participants Twenty-two healthy pregnant women participated in this study. Gestational age ranged from 27 to 36 weeks (mean 30.9 weeks). Informed consent was received from the participants before any measurements were made. The Ethical Committee of the Medical Faculty of the University of Tübingen approved the study plan.

Of the 22 participants, four had to discontinue the study due to fatigue and data analysis was not possible for a further five due to weak fetal signals, maternal muscle activity or movement artefacts. Therefore, data from 13 women was analysed. All pregnancies were uncomplicated singleton pregnancies according to maternal pregnancy record books. None of the fetuses were macrosomic at birth. Two out of 13 women declared smoking up to five cigarettes a day.

Paradigm All measurements were started at 08:00 hours at the fMEG Center at the University of Tübingen. After an overnight fast of at least $5 \mathrm{~h}$, each participant ingested a solution containing $75 \mathrm{~g}$ glucose (Accu-Chek Dextrose O.G-T.; Roche Diagnostics, Germany). Venous blood samples were obtained before ingestion of the glucose solution ( $0 \mathrm{~min})$ and after 60 and $120 \mathrm{~min}$. In one participant, a venous blood sample could not be obtained at $60 \mathrm{~min}$. Blood glucose and plasma insulin concentrations were determined in the laboratory.

Each blood extraction was preceded by an fMEG measurement. The baseline fMEG measurement was performed before the baseline blood extraction. The second and third fMEG measurement was started after 50 and $110 \mathrm{~min}$, respectively.

Before the first and after the last fMEG measurement an ultrasound scan was performed to determine the fetal head position. Between measurements, the medical history of the participants was recorded and cardiotocography (CTG) was performed. The relevant steps of the paradigm are shown in Table 1.

Laboratory measurements and calculations Blood glucose concentrations were determined using a glucose analyser (glucose oxidase method; Yellow Springs Instruments, Yellow Springs, OH, USA). Plasma insulin was analysed using the ADVIA Centaur XP immunoassay system (Siemens AG, Erlangen, Germany). Insulin resistance was calculated by means of the HOMA-IR [24]. For better characterisation of the postprandial insulin sensitivity, the following index was

Table 1 Paradigm

\begin{tabular}{|c|c|c|}
\hline $\begin{array}{l}\text { Measurement } \\
\text { time point }\end{array}$ & $\begin{array}{l}\text { Timing } \\
(\mathrm{min})\end{array}$ & Procedure \\
\hline & & Ultrasound scan \\
\hline \multirow[t]{2}{*}{1} & & First auditory fMEG measurement (baseline) \\
\hline & & $\begin{array}{l}\text { Laying of venous access } \\
\text { First blood extraction (baseline) }\end{array}$ \\
\hline \multirow[t]{3}{*}{2} & 0 & Drinking OGTT solution \\
\hline & $50+$ & Second auditory fMEG measurement \\
\hline & 60 & Second blood extraction \\
\hline \multirow[t]{4}{*}{3} & $110+$ & Third auditory fMEG measurement \\
\hline & 120 & Third blood extraction \\
\hline & & Ultrasound scan \\
\hline & & Breakfast \\
\hline
\end{tabular}


calculated (as recommended by Stumvoll et al [25]) for a three-point OGTT in units of $\mu \mathrm{mol} \mathrm{kg}{ }^{-1} \mathrm{~min}^{-1} \mathrm{pmol} / \mathrm{l}$ :

$$
\begin{aligned}
0.156 & -0.0000459 \times \mathrm{Ins}_{120 \mathrm{~min}}-0.000321 \times \mathrm{Ins}_{0 \mathrm{~min}}-0.00541 \\
\times & \times \text { Gluc }_{120 \mathrm{~min}}
\end{aligned}
$$

fMEG measurement $\mathrm{fMEG}$ data were recorded with the SARA system (SQUID Array for Reproductive Assessment, VSM MedTech, Port Coquitlam, BC, Canada) installed at the fMEG Center at the University Tübingen. In this system biomagnetic signals generated by electrical currents in the body are recorded with 156 primary magnetic sensors, which are distributed over a concave array, shaped to match the form of the gravid abdomen.

Based on the first ultrasound measurement (Ultrasound Logiq 500MD; GE Healthcare, Pollards Wood, UK), the position of fetal head was marked by a localisation coil placed directly on the abdomen. This location was reconfirmed via ultrasound after the last measurement. Three additional localisation coils were placed on the mother's spine and on her left and right side. At the beginning and end of each recording, localisation coils were activated at a certain frequency to determine their coordinates in relation to the sensors. Data were recorded with a sampling rate of $610.352 \mathrm{~Hz}$.

During each fMEG measurement, an auditory sequence was presented for $6 \mathrm{~min}$ and the evoked fetal brain activity was recorded.

Auditory stimulation procedure Two tones were presented in an auditory oddball paradigm. A frequent 'standard' tone with a frequency of $500 \mathrm{~Hz}$ was presented $75 \%$ of the time. This was randomly interspersed with an infrequent 'deviant' tone (frequency of $750 \mathrm{~Hz}$, presented $25 \%$ of the time) to prevent habituation to the standard tone. Tone duration was $500 \mathrm{~ms}$, tone intensity was $95 \mathrm{~dB}\left(3.2 \times 10^{-3} \mathrm{~W} / \mathrm{m}^{2}\right)$. Attenuation of the sound intensity by maternal tissue implies that tones with an intensity of about $65 \mathrm{~dB}\left(3.2 \times 10^{-6} \mathrm{~W} / \mathrm{m}^{2}\right)$ reach the fetus [26]. Stimuli were generated as tone bursts with $10 \mathrm{~ms}$ rise and fall times. The inter-stimulus interval was set to $1,500 \mathrm{~ms}$. Stimulus delivery was controlled using the Presentation program (version 12.2, www.neurobs.com). The sound was generated by a speaker and transmitted by means of plastic tubing to an inflated plastic bag (height approximately $3 \mathrm{~cm}$ ) placed between the sensor array and maternal abdomen. Reported results refer to the standard tone.

Fetal data analysis Data were collected in continuous mode. Maternal and fetal heart signals were attenuated with standard algorithms [27, 28]. A bandpass filter between 0.5 and $10 \mathrm{~Hz}$ was applied to the data. Data were split into trials based on stimulus type (200 ms before, 1,000 ms after stimulus onset).
Trials containing amplitudes above $2 \mathrm{pT}$ were rejected. Trials were then averaged according to stimulus type.

Evoked responses (ERs) to the standard tone were identified by visual inspection. The evoked activity had to be located in the area near the position of the head coil [29]. The five channels with the highest amplitude of the ER were selected and latencies were determined by the peak root mean square amplitude of those channels.

Statistical analysis Statistical tests were performed with SPSS (version 20.0; IBM SPSS Statistics for Windows, Armonk, NY, USA) and results with $p<0.05$ were regarded as statistically significant. Missing values were excluded pairwise.

A repeated measures ANOVA of measurement time point (oral glucose) on ER latency was performed. Additionally, paired $t$ tests were used to compare variables between time points. Since a possible effect on ER latencies could be caused by a change in either maternal blood glucose or plasma insulin level, linear regressions analyses were performed to examine the influence of glucose and insulin levels on response latencies of the fetus for the different time points.

For the second measurement time point, Pearson correlations were used to explore the connection between maternal insulin sensitivity and fetal auditory response latency. Response latencies between two insulin sensitivity subgroups were compared with two-sample $t$ tests.

\section{Results}

Participants Data from 13 pregnant healthy women were included in this study. The metabolic and clinical characteristics of the participants are shown in Table 2.

Table 2 Characteristics of the study participants

\begin{tabular}{ll}
\hline Characteristic & Value \\
\hline$n$ & 13 \\
Age (years) & $32 \pm 3$ \\
Duration of pregnancy (weeks) & $31 \pm 3$ \\
BMI before pregnancy $\left(\mathrm{kg} / \mathrm{m}^{2}\right)$ & $23.6 \pm 3.3$ \\
Absolute weight gain during pregnancy $(\mathrm{kg})$ & $10.0 \pm 2.2$ \\
Relative weight gain during pregnancy $(\mathrm{kg} /$ week $)$ & $0.32 \pm 0.07$ \\
Fasting glucose $(\mathrm{mmol} / \mathrm{l})$ & $4.4 \pm 0.3$ \\
2 h glucose (mmol/l) & $6.4 \pm 1.1$ \\
Fasting insulin (pmol/l) & $67 \pm 25$ \\
2 h insulin (pmol/l) & $792 \pm 368$ \\
HOMA-IR & $1.9 \pm 0.7$ \\
Insulin sensitivity index $\left(\mu \mathrm{mol} \mathrm{kg}{ }^{-1} \mathrm{~min}^{-1} \mathrm{pmol} / \mathrm{l}\right)$ & $0.063 \pm 0.026$ \\
\hline
\end{tabular}

Data are presented as means $\pm \mathrm{SD}$ 
Auditory measurements Fetal auditory evoked responses (AERs) could be analysed for 13 participants. For two participants, no AER was detectable at time point 1 , for a further two participants, no AER was detectable at time point 2 .

Table 3 shows mean AER latencies to standard tones, as well as maternal blood glucose and plasma insulin levels for all three measurement time points, including values from all participants.

As expected during an oral glucose challenge, maternal blood glucose and plasma insulin levels increased from baseline to $60 \mathrm{~min}$ and decreased again at $120 \mathrm{~min}$. There was also a change in fetal ER latency during the course of the oral glucose challenge. A repeated measures ANOVA revealed a significant main effect of the oral glucose challenge on AER latency $(F[2]=4.8, p=0.024)$. Response latency was shortened during the OGTT from a fasting level at 0 to $60 \mathrm{~min}$ after glucose administration $(297 \pm 99$ vs $235 \pm 84 \mathrm{~ms}, t[8]=$ $3.3, p=0.01)$. There was no significant change in response latency between 60 and $120 \mathrm{~min}(235 \pm 84$ vs $251 \pm 91 \mathrm{~ms}$, $t[10]=0.9, p=0.39)$. Linear regression analysis revealed an association between maternal insulin level and the ER of the fetus at $60 \mathrm{~min}$ after glucose ingestion $(F[1]=5.45, p=0.05)$. There was no significant effect of maternal insulin level at 0 and $120 \mathrm{~min}$ and maternal glucose level at 0,60 and $120 \mathrm{~min}$ on corresponding ERs.

There was a significant correlation between maternal insulin sensitivity and response latency of the fetus $(r=0.68$, $p=0.02$ ) $60 \mathrm{~min}$ after ingestion of glucose: the higher the insulin sensitivity of the mother, the shorter was the response latency of the fetus (Fig. 1). The correlation remained significant after controlling for relative weight gain of the mother, gestational age or weight of the child at birth.

There was no significant correlation between maternal insulin sensitivity index and response latency of the fetus at baseline or after $120 \mathrm{~min}(r=0.03, p=0.93$ and $r=0.21$, $p=0.48$, respectively).

The women were split into two groups based on the median of the insulin sensitivity index - insulin resistant $\left(0.081 \pm 0.023 \mu \mathrm{mol} \mathrm{kg}^{-1} \mathrm{~min}^{-1} \mathrm{pmol} / \mathrm{l}\right)$ and insulin sensitive $\left(0.043 \pm 0.008 \mu \mathrm{mol} \mathrm{kg}{ }^{-1} \mathrm{~min}^{-1} \mathrm{pmol} / \mathrm{l}\right)$. The insulin sensitivity index differed significantly between groups $(t[8]=4.1$, $p=0.004)$. The response latency of fetuses $60 \mathrm{~min}$ after

Table 3 Maternal blood glucose and plasma insulin levels and corresponding ER latencies to standard tones for all three measurements

\begin{tabular}{llll}
\hline Variable & \multicolumn{3}{l}{ Measurement time point } \\
\cline { 2 - 4 } & $1(0 \mathrm{~min})$ & $2(60 \mathrm{~min})$ & $3(120 \mathrm{~min})$ \\
\hline ER latency to standard tone $(\mathrm{ms})$ & $297 \pm 99$ & $235 \pm 84$ & $251 \pm 91$ \\
Insulin (pmol/l) & $67 \pm 25$ & $918 \pm 492$ & $792 \pm 368$ \\
Glucose (mmol/l) & $4.4 \pm 0.3$ & $7.4 \pm 1.1$ & $6.4 \pm 1.1$ \\
\hline
\end{tabular}

Data are presented as means $\pm \mathrm{SD}, n=13$

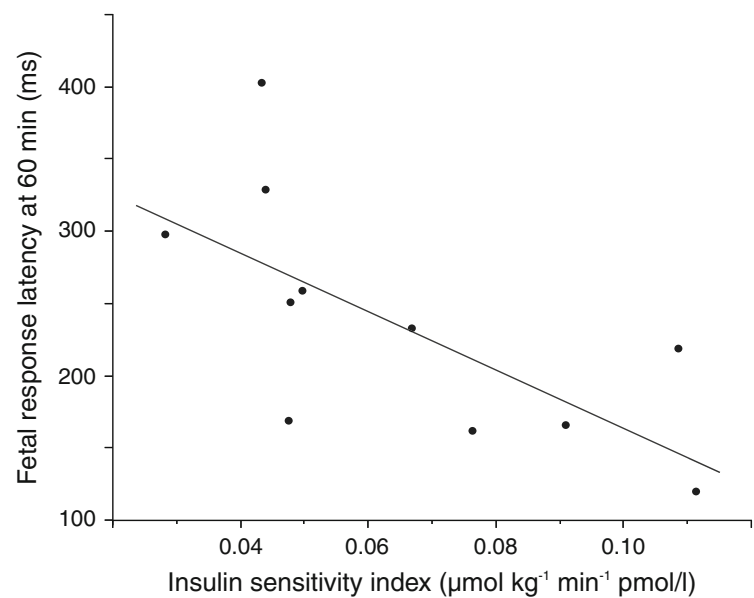

Fig. 1 Maternal insulin sensitivity index and fetal response latency 60 min after glucose ingestion

glucose ingestion by insulin-resistant mothers was delayed in comparison with the response latency of fetuses of insulin-sensitive mothers $(283 \pm 79$ vs $178 \pm 46 \mathrm{~ms}, t[9]=2.6$, $p=0.03$ ). Figure 2 shows mean maternal glucose and insulin levels and mean fetal response latencies during the OGTT in both insulin-resistant and insulin-sensitive groups. There was
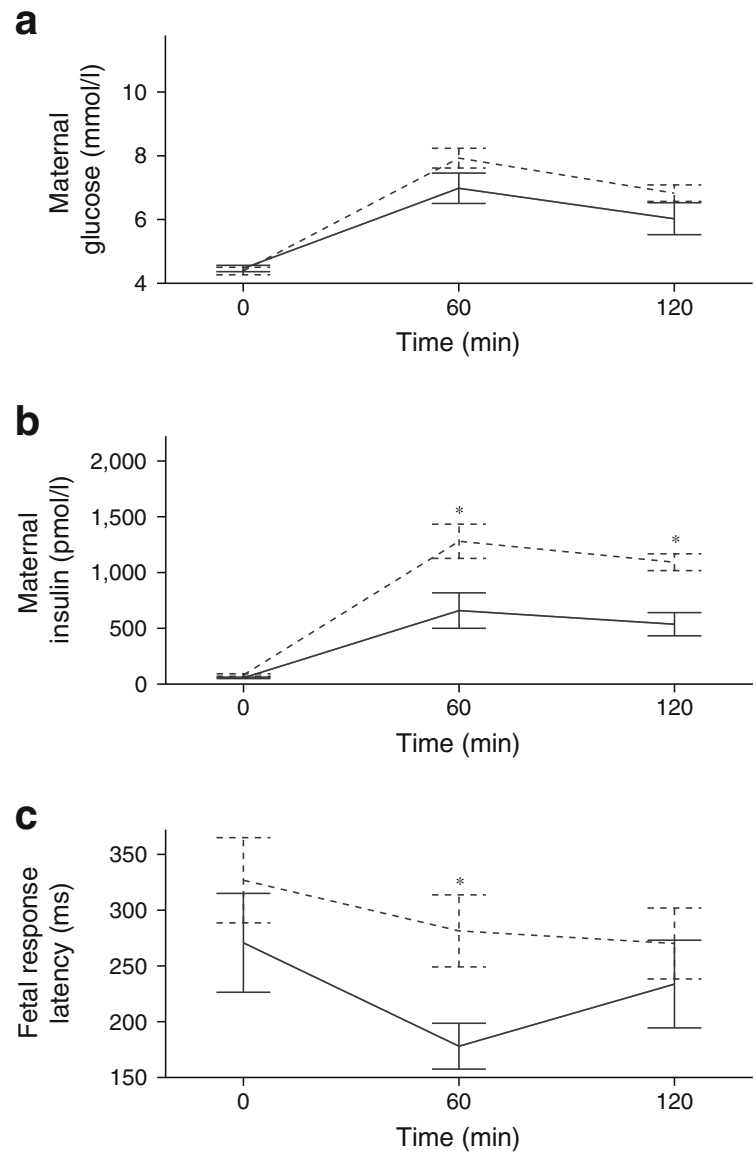

Fig. 2 Maternal glucose levels (a), maternal insulin levels (b) and fetal response latencies (c) during OGTT in insulin-resistant ( $n=6$, dashed line) and insulin-sensitive women ( $n=7$, solid line). Data are shown as mean \pm SEM; ${ }^{*} p<0.05$ between groups 
a significant difference in insulin level between groups after $60 \min (t[10]=2.71, p=0.02)$ and after $120 \min (t[11]=4.19$, $p=0.002$ ).

A similar association was observed when maternal insulin sensitivity was calculated from fasting glucose and insulin levels using HOMA-IR. Insulin-sensitive and -resistant women differed significantly in HOMA-IR $(1.29 \pm 0.34$ vs $2.44 \pm$ $0.51, t[11]=-4.69, p=0.001)$. The HOMA-IR of mothers and response latency of fetuses $60 \mathrm{~min}$ after ingestion of glucose by the mother correlated significantly $(r=0.71, p=0.01)$; the response latency was delayed in fetuses of insulin-resistant mothers when compared with fetuses of insulin-sensitive mothers $(281 \pm 81$ vs $179 \pm 45 \mathrm{~ms}, t[9]=2.5, p=0.03)$.

\section{Discussion}

In the present study, we showed for the first time that fetal brain activity is altered in the postprandial state during an oral glucose challenge and also that maternal insulin sensitivity is associated with fetal brain activity. In particular, we showed that $60 \mathrm{~min}$ after glucose ingestion, fetuses of pregnant woman with lower insulin sensitivity (insulin-resistant group) have longer response latencies than fetuses of pregnant woman with higher insulin sensitivity (insulin-sensitive group).

AERs in fetuses, recorded using fMEG, have previously been demonstrated $[18,22]$ and the latencies of AERs decreasing with gestational age have been interpreted as an indicator of brain maturation. A recent study showed that intrauterine growth restriction caused by placental insufficiency leads to an increase in the latency of fetal AERs with a similar paradigm [23]. Both of these findings clearly show that the nutrient supply to the fetus has an effect on fetal brain activity. Interestingly the current study showed a differential effect of the OGTT for insulin-sensitive and -resistant pregnant women on fetal functional brain activation in the postprandial maternal state.

Speculation concerning the mechanisms for this differential effect can be based on the findings shown in Fig. 2 (visualised in Fig. 3), demonstrating that insulin-resistant mothers have higher glucose levels accompanied by increased insulin levels in the postprandial state. As glucose passes the placenta, these postprandially increased glucose levels induce hyperinsulinaemia in the fetus (Pedersen hypothesis [30]). Therefore, high insulin levels in the mother may correspond to high insulin levels in the fetus. It is possible that high insulin levels are a prerequisite for appropriate brain maturation. However, chronic hyperinsulinaemia, which is present in insulin-resistant mothers and corresponds to high insulin levels in the fetus, might induce insulin resistance in the fetal brain. Of course, there are several other possible mechanisms explaining the association between the change in response

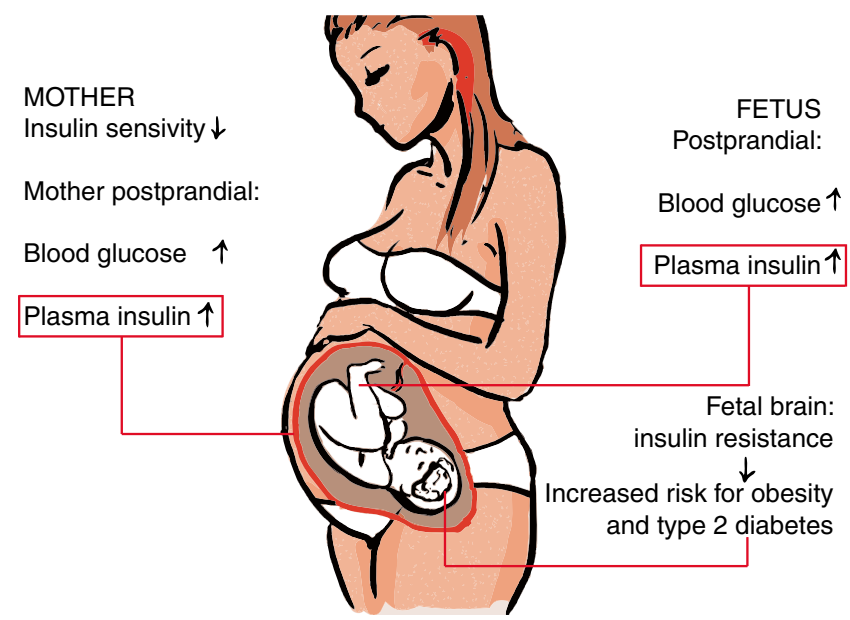

Fig. 3 Possible pathogenesis of fetal brain insulin resistance

latency in the fetal brain and the insulin sensitivity of the mother: the insulin resistance of the mother may be associated with a limited insulin transport into the fetal brain; alternatively, the insulin resistance of the mother may be associated with a variety of other hormonal and metabolic fuel effects, which actually mediate the change in response latency [31]. Therefore, at present we can only speculate that the high insulin levels in the fetus associated with maternal insulin resistance induce primary insulin resistance of the fetal brain.

The insulin resistance of the fetal brain may be interpreted as metabolic imprinting of insulin resistance with important consequences for later life. The consequent effect of hyperinsulinaemia on fetal development has already been shown. Compared with newborns of non-diabetic women, children of diabetic mothers with poorly controlled glycaemia show neurophysiological impairment and have a higher risk for metabolic syndrome, obesity and type 2 diabetes mellitus in later life $[15,16,32]$.

The assumption that an insulin-resistant fetal brain may have an impact on the development of metabolic syndrome, obesity and type 2 diabetes mellitus in later life is supported by recent studies investigating the effect of impaired insulin action on the central nervous system in adults. The action of insulin in the brain is thought to control metabolism and behaviour, and insulin resistance of the brain may be associated with obesity, diabetes and cognitive impairment [1]. For example, we recently demonstrated cross-sectionally that a reduced cerebrocortical response to insulin during a hyperinsulinaemic clamp, in overweight compared with lean humans, is associated with obesity and increased NEFA [7, 8]. Furthermore, insulin resistance of the brain prospectively predicts less weight reduction in individuals taking part in lifestyle intervention programmes [6].

A limitation of the present study is the small number of participants. Additionally, direct measurement of insulin levels in the amniotic fluid would have been desirable, although this was not possible for ethical reasons. The 
gestational age in the present study varied between 28 and 36 weeks. According to previous fMEG studies, ER latencies seem to decrease with fetal age, and are interpreted as an indicator of brain maturity. Since our results remained significant after adjustment for gestational age, it is unlikely that the increased response latency is caused by differing gestational age.

In summary, our study showed that fetal brain activity increases postprandially, as indicated by shorter fetal response latencies to auditory stimuli. Furthermore, the level of maternal insulin resistance affects the fetal brain activity in postprandial conditions. This finding constitutes important evidence supporting the fetal programming theory. Central insulin resistance could already be developed in utero. Future investigations will further examine the effects that pathological insulin resistance and chronic exposure to higher insulin levels in diabetic mothers have on fetuses and children.

Acknowledgements The authors acknowledge the assistance of M. Weiss (fMEG Center, University of Tübingen) with data collection and the assistance of M.-A. Schneider (Department of Internal Medicine, Division of Endocrinology, Diabetology, Angiology, Nephrology and Clinical Chemistry, University Hospital Tübingen) with proofreading the manuscript.

Funding This work was partially funded by a grant from the German Federal Ministry of Education and Research (BMBF) to the German Center for Diabetes Research (DZD e.V.), by the Helmholtz Alliance ICEMED - Imaging and Curing Environmental Metabolic Diseases, through the Initiative and Network Fund of the Helmholtz Association and German Research Foundation (TR-SFB 654 "Plasticity and Sleep").

Duality of interest The authors declare that there is no duality of interest associated with this manuscript.

Contribution statement KL and FS contributed equally to this work, were involved in the design of the study, data acquisition and analysis and interpretation of data and drafted and revised the article. CK contributed to the study design and revised the article. LF and IKS were involved in data acquisition and revised the article. AH contributed to the conception and design of the study and revised the article. HUH contributed to interpretation of the data and revised the article. HP and AF contributed to the conception and design of the study and to the analysis and interpretation of data and drafted and revised the article. All authors approved the current version of the article. $\mathrm{HP}$ and $\mathrm{AH}$ are the guarantors of this article.

\section{References}

1. Hallschmid M, Schultes B (2009) Central nervous insulin resistance: a promising target in the treatment of metabolic and cognitive disorders? Diabetologia 52:2264-2269

2. Plum L, Belgardt BF, Bruning JC (2006) Central insulin action in energy and glucose homeostasis. J Clin Invest 116:1761-1766

3. Porte D, Baskin DG, Schwartz MW (2005) Insulin signaling in the central nervous system: a critical role in metabolic homeostasis and disease from C. elegans to humans. Diabetes 54:1264-1276
4. Benedict C, Brede S, Schiöth HB et al (2011) Intranasal insulin enhances postprandial thermogenesis and lowers postprandial serum insulin levels in healthy men. Diabetes 60:114-118

5. Heni M, Kullmann S, Ketterer C et al (2012) Nasal insulin changes peripheral insulin sensitivity simultaneously with altered activity in homeostatic and reward-related human brain regions. Diabetologia 55:1773-1782

6. Tschritter O, Preissl H, Hennige AM et al (2012) High cerebral insulin sensitivity is associated with loss of body fat during lifestyle intervention. Diabetologia 55:175-182

7. Tschritter O, Preissl H, Hennige AM et al (2006) The cerebrocortical response to hyperinsulinemia is reduced in overweight humans: a magnetoencephalographic study. Proc Natl Acad Sci U S A 103: 12103-12108

8. Tschritter O, Preissl H, Hennige AM et al (2009) The insulin effect on cerebrocortical theta activity is associated with serum concentrations of saturated nonesterified fatty acids. J Clin Endocrinol Metab 94: 4600-4607

9. Nolan CJ, Damm P, Prentki M (2009) Type 2 diabetes across generations: from pathophysiology to prevention and management. Lancet 378:169-181

10. Plagemann A (2008) A matter of insulin: developmental programming of body weight regulation. J Matern Fetal Neonatal Med 21: 143-148

11. Barker DJ, Osmond C, Simmonds SJ, Wield GA (1993) The relation of small head circumference and thinness at birth to death from cardiovascular disease in adult life. BMJ 306:422-426

12. Dabelea D, Crume T (2011) Maternal environment and the transgenerational cycle of obesity and diabetes. Diabetes 60:1849-1855

13. Rao S, Yajnik CS, Kanade A et al (2001) Intake of micronutrient-rich foods in rural Indian mothers is associated with the size of their babies at birth: Pune Maternal Nutrition Study. J Nutr 131:1217-1224

14. Yajnik CS (2004) Early life origins of insulin resistance and type 2 diabetes in India and other Asian countries. J Nutr 134:205-210

15. Schaefer-Graf UM, Pawliczak J, Passow D et al (2005) Birth weight and parental BMI predict overweight in children from mothers with gestational diabetes. Diabetes Care 28:1745-1750

16. Sobngwi E, Boudou P, Mauvais-Jarvis F et al (2003) Effect of a diabetic environment in utero on predisposition to type 2 diabetes. Lancet 361:1861-1865

17. Catalano PM, Presley L, Minium J, Mouzon SH (2009) Fetuses of obese mothers develop insulin resistance in utero. Diabetes Care 32: 1076-1080

18. Preissl H, Lowery CL, Eswaran H (2004) Fetal magnetoencephalography: current progress and trends. Exp Neurol 190(Suppl 1):28-36

19. Eswaran H, Wilson JD, Preissl H et al (2002) Magnetoencephalographic recordings of visual evoked brain activity in the human fetus. Lancet 360:779-780

20. Eswaran H, Preissl H, Wilson JD et al (2002) Short-term serial magnetoencephalography recordings of fetal auditory evoked responses. Neurosci Lett 331:128-132

21. Schneider U, Schleussner E, Haueisen J, Nowak H, Seewald HJ (2001) Signal analysis of auditory evoked cortical fields in fetal magnetoencephalography. Brain Topogr 14:69-80

22. Holst M, Eswaran H, Lowery C, Murphy P, Norton J, Preissl H (2005) Development of auditory evoked fields in human fetuses and newborns: a longitudinal MEG study. Clin Neurophysiol 116: 1949-1955

23. Kiefer ID, Siegel ER, Preissl H et al (2008) Delayed maturation of auditory evoked responses in growth-restricted fetuses revealed by magnetoencephalographic recordings. Am J Obstet Gynecol 199: 503.e1-503.e7

24. Matthews DR, Hosker JP, Rudenski AS, Naylor BA, Treacher DF, Turner RC (1985) Homeostasis model assessment: insulin resistance and beta-cell function from fasting plasma glucose and insulin concentrations in man. Diabetologia 28:412-419 
25. Stumvoll M, van Haeften T, Fritsche A, Gerich J (2001) Oral glucose tolerance test indexes for insulin sensitivity and secretion based on various availabilities of sampling times. Diabetes Care 24:796-797

26. Querleu D, Renard X, Versyp F, Paris-Delrue L, Crèpin G (1988) Fetal hearing. Eur J Obstet Gynecol Reprod Biol 29:191-212

27. McCubbin J, Robinson SE, Cropp R et al (2006) Optimal reduction of MCG in fetal MEG recordings. IEEE Trans Biomed Eng 53:1720 1724

28. Vrba J, Robinson SE, Mccubbin J et al (2004) Fetal MEG redistribution by projection operators. IEEE Trans Biomed Eng 51:12071218
29. Vrba J, Robinson SE, McCubbin J et al (2004) Human fetal brain imaging by magnetoencephalography: verification of fetal brain signals by comparison with fetal brain models. NeuroImage 21:1009-1020

30. Pedersen J (1967) The pregnant diabetic and her newborn: problems and management. William \& Wilkins, Baltimore

31. Catalano PM, Hauguel-De Mouzon S (2011) Is it time to revisit the Pedersen hypothesis in the face of the obesity epidemic? Am J Obstet Gynecol 204:479-487

32. Silverman BL, Metzger BE, Cho NH, Loeb CA (1995) Impaired glucose tolerance in adolescent offspring of diabetic mothers. Relationship to fetal hyperinsulinism. Diabetes Care 18:611-617 